\title{
Study of Longevity, Fecundity and Oviposition of Trichogrammatoidea bactrae Nagaraja (Hymenoptera: Trichogrammatidae) to Facilitate Mass Rearing
}

\author{
M.C.D. Perera ${ }^{*}$ and K.S. Hemachandra ${ }^{1}$ \\ Postgraduate Institute of Agriculture \\ University of Peradeniya \\ Sri Lanka
}

\begin{abstract}
Trichogrammatoidea bactrae (Nagaraja) has been identified as a potential egg parasitoid for managing rice leaf folder (Cnaphalocrocis medinalis) in rice (Oryza sativa L.). Different sugars, i.e. saturated solutions of glucose, fructose, sucrose, undiluted bee honey and distilled water were evaluated as diets for the adult parasitoids in terms of adult longevity. There was a significant variation of longevity among the adults fed with different feed sources. Parasitoids fed with undiluted honey lived the longest $(7.2 \pm 0.3$ days). Subsequently, bee honey at different concentrations, i.e. 100, 50, 33, 25, 20 and 17\% was evaluated in terms of adult longevity. The highest longevity of $7.9 \pm 0.2$ days was found when parasitoid adults were fed with $50 \%$ bee honey while the lowest longevity was $1.5 \pm 0.2$ days on distilled water. Several standard sugar feeding methods were also evaluated in terms of longevity. Honey feeding by filter paper method was the most suited compared to other methods i.e. honey drop, sponge and wick methods. With respect to fecundity, the rate of oviposition with adult age was examined. There was a significant variation of daily oviposition with age. One-day-old parasitoids laid $31.2 \pm 3.27$ eggs per female with $56.05 \%$ of total fecundity, and the laying was reduced to 0.4 eggs per female in five days old parasitoids. The mean fecundity was $55.7 \pm 0.26$ eggs per female. Effect of rearing of parasitoids on alternate hosts (Corcyra cephalonica) on oviposition was significant. There was a general trend of loosing the ability of parasitism with continuous rearing of progeny on alternative host.
\end{abstract}

Keywords: Fecundity, food sources, longevity, parasitism, Trichogrammatoidea bactrae

\section{INTRODUCTION}

Integrated pest management (IPM) has been widely accepted as an option to minimize the use of insecticides in agro ecosystems. Biological control of insect pests has been recognized as a strong component of IPM considering the sustainability and environment friendliness. Among the biocontrol approaches, augmentative release has been suggested considering the safety and efficacy. In this process, biocontrol agents are reared in the laboratory and released to target sites in large numbers for suppression of pest populations. Among the parasitoids, egg parasitoids have special advantages over larval or pupal parasitoids because, egg parasitoids destroy the pest species before it attacks the crop. Around the globe, 91 egg parasitoid species have been used for the control of 77 different pest species (Greathead \& Greathead, 1992). In order to attempt biological control of rice leaf folder, Cnaphalocrocis medinalis (Guen.) (Lepidoptera: Pyralidae) using egg parasitoids, it is necessary to develop a

\footnotetext{
1 Department of Agricultural Biology, Faculty of Agriculture, University of Peradeniya, Sri Lanka

* Corresponding author: pereramcd@gmail.com
} 
protocol for mass rearing of egg parasitoids. In that respect, it is necessary to keep the egg parasitoids alive giving the best suited adult food, in a best suited way. In addition, reproductive biology of parasitoids with respect to fecundity and longevity should be studied to obtain maximum production of adult parasitoids.

The main energy source required by adult parasitoids is sugar and parasitoids naturally obtain sugar from flower nectar in the field or from honeydew (Hagley \& Barber, 1992). The quantity and quality of sugar sources in the field vary as they can be obtained from various sources. Sugar concentration in nectar also varies even within one flower over time and even within the course of one day (Mohr \& Jay, 1990).

Many species of parasitic wasps can increase their reproduction through feeding on supplementary sugar sources. Carbohydrate source affects longevity of adult parasitoids (Jacob \& Evans, 1998). Sugar feeding usually results in an increase in longevity that is often positively correlated with a higher fecundity (Hagley \& Barber, 1992). Laboratory and field cage studies demonstrate that parasitoids that have access to sugar have greater longevity, fecundity, and more female biased sex ratios than starved parasitoids (Idris \& Grafius, 1995).

In many other countries, several lepidopteron pests have been managed through augmentative release of Trichogramma species, the egg parasitoids (Elzen \& King, 1999). Effective biological control through augmentation and release of parasitoids requires a substantial knowledge of the parasitoid biology. As the first step in evaluating the potential of using egg parasitoids to manage rice leaf folder (C. medinalis), a colony of egg parasitoid species, Trichogrammatoidea bactrae was established. Laboratory tests were carried out to evaluate the potential of the T. bactrae as a potential biological control agent for the rice leaf folder and found promising results.

Hence, our objectives in this study were to determine the best source of sugar for parasitoids in terms of longevity, the best concentration of sugar suitable for parasitoids and the best method of feeding parasitoids. In addition, parasitoid performance was evaluated along with number of generation in rearing cultures.

\section{METHODOLOGY}

\section{Insects and experimental preparation}

Trichogrammatoidea bactrae was used as the parasitoid species in the study. It was identified in previous studies as a potential parasitoid species that could be used in managing rice leaf folder, $C$. medinalis. A culture of $T$. bactrae was established by collecting $C$. medinalis eggs in rice fields in Peradeniya area. The collected eggs were individually reared in plastic vials and emerged parasitoids were used to establish the culture. The culture was continued using the alternate host, Corcyra cephalonica which was reared on maize grains. The experiments were conducted under room temperature $\left(27{ }^{\circ} \mathrm{C}, 65 \% \mathrm{RH}\right)$.

\section{Assessment of suitability of different food type}

It has been established that different sugars have different effects on parasitoids depending on the species. There were six treatments and ten replicates. Altogether hundred individuals of parasitoids were fed with each food type and longevity was recorded for each individual separately. The selected sugar sources used for this study were glucose, fructose, sucrose and 
undiluted honey. Glucose, fructose, and sucrose were used as a saturated solution. Ten newly emerged parasitoid adults (male and female) were enclosed in a clear plastic vial $(4.3 \mathrm{~cm}$ diameter and $5.5 \mathrm{~cm}$ high) and food was supplied. This was replicated ten times. Food was provided placing a drop of each sugar solution on inner side of lid of the plastic vials. Ten newly emerged parasitoids were maintained without food in a vial, replicated ten times and considered as control, and another set with similar numbers was maintained only with distilled water. Vials were examined everyday and recorded the mortality and the longevity was calculated. The method was adapted according to Saljoqi \& Khajjak, (2007).

\section{Evaluation of honey concentration suitable for adult parasitoids}

In the previous experiment, bee honey was proved as the best type of food. Different concentrations of bee honey were evaluated in this experiment. Pure undiluted honey was diluted as 1:1, 1:2, 1:3, 1:4, 1:5, honey: water solutions. Concentration was 50, 33, 25, 20, and $17 \%$ respectively. Distilled water was used as the control. Pure honey was also included in the experiment. Honey solutions were provided as follows. Six different honey solutions were placed in separate containers and portion of filter paper (1/16 of a filter paper (9 $\mathrm{cm}$ diameter)) was dipped for 30 minutes for better absorption of honey. Subsequently, excess honey was drained out and the filter paper was placed in the plastic transparent vial $(4.3 \mathrm{~cm}$ diameter and $5.5 \mathrm{~cm}$ high) containing one day old parasitoids. There were seven treatments and ten replicates. Each replicate consisted with ten one-day-old parasitoids, male and female in a vial. Parasitoids fed with distilled water were considered as the control. Mortality of the parasitoids was examined everyday and the longevity was calculated. Other details of the experiment were similar to experiment one and adapted from Azzouza et al., (2004).

\section{Evaluation of different methods of feeding of adult parasitoids}

In this study, four commonly used methods of adult feeding were tested. There were four treatments and eight replicates. Each replicate has ten adult parasitoids. In the first method, a filter paper (1/16 part of filter paper) was soaked in 30 minutes and excess pure honey was allowed to drain, subsequently used for adult feeding. In the second feeding method, a piece of sponge $(1 \times 1 \times 1 \mathrm{~cm})$ was soaked in $100 \%$ honey solution and excess honey was allowed to drain and used for adult feeding. In the third feeding method, a cotton wick $(1.5 \mathrm{~cm}$ long) was dipped in 100 percent honey for 30 minutes and used after draining excess honey solution. Food source under different feeding techniques was placed in separate transparent plastic vials $(4.3 \mathrm{~cm}$ diameter and $5.5 \mathrm{~cm}$ high) having ten one-day-old parasitoids and closed the lid. At the same time, one drop of each solution put in to bottom of separate transparent plastic containers $(4.3 \mathrm{~cm}$ diameter and $5.5 \mathrm{~cm}$ high) having ten parasitoids of one-day-old and closed the lid. The control consisted with unfed parasitoids. Each treatment was replicated eight times. The rearing containers were checked for dead parasitoids in the morning in each day until the last adult parasitoid die.

\section{Determination of the fecundity of the parasitoids}

In order to determine the fecundity, the newly emerged, eight mated female parasitoid was enclosed in a separate plastic vial $(4.3 \mathrm{~cm}$ diameter and $5.5 \mathrm{~cm}$ high) containing 80 , one-dayold $C$. cephalonica eggs, prepared as an egg card. Undiluted honey was placed inside the lid of each vial as a drop and the vial was closed. Each vial was held in natural environmental conditions $\left(65 \% \mathrm{RH}\right.$ and $\left.27^{\circ} \mathrm{C}\right)$. After every 24 hours, egg card was replaced with new egg card, which has 80 C. cephalonica eggs. This procedure was continued up to the point no 
more oviposition was observed. The removed eggs cards were continuously observed. When eggs turn black, the numbers of parasitized eggs were counted.

\section{Determining parasitism levels over different generations}

It has been reported that the egg parasitoids loose the capacity of egg laying when they were reared continuously on alternative host eggs. This effect was studied in this experiment, using T. bactrae. Newly emerged (one-day-old) single female parasitoid was placed in a separate transparent plastic vial $(4.3 \mathrm{~cm}$ diameter and $5.5 \mathrm{~cm}$ high) having one-day-old $C$. cephalonica egg card. Each egg card had 100 of C. cephalonica eggs. Undiluted honey was supplied as food. Nine mated female parasitoids were used for the experiment. After five days, number of parasitized eggs was counted. After that, one day old adult females emerged from parasitized eggs were enclosed with one-day-old $C$. cephalonica egg cards and again number of parasitized eggs were counted. Same procedure was followed up to fifteen generations.

\section{Data Analysis}

Data were analyzed using ANOVA in SYSTAT 13 version. Means were separated using LSD.

\section{RESULTS AND DISCUSSION}

\section{Assessment of suitability of different food types}

The longevity is an important character of adult parasitoids; that affect on oviposition and eventually the overall production of mass culture. The longevity depends on food sources which were obtained in environment; mostly the flower nectar or homopteran honey dew. These sources contain different concentration of honey and the sugar composition also varies among sources (Lee \& Heimpel, 2005).

There was a significant variation of longevity of $T$. bactrae adults when they were fed on different food types $(\mathrm{F}=8.229 \mathrm{df}=5,574 \mathrm{P}<0.001)$. Parasitoids lived significantly longer period of time when they were fed on bee honey $(7.2 \pm 0.3 \mathrm{~d})$ than glucose $(7.0 \mathrm{~d})$, sucrose $(4.9 \mathrm{~d})$, fructose $(4.7 \mathrm{~d})$ and distilled water $(2.1 \mathrm{~d})$ days respectively. The parasitoids who did not receive any food had the shortest lifespan, $1.7 \pm 0.3$ days.

A similar result was found in other studies conducted with different parasitoid species (Saljoqi \& Khajjak, 2007). Honey appears to be a good source of food for adult parasitoids; Catolaccus grandis (Burks) (Hymenoptera: Pteromalidae) lived a significantly longer period when they were fed on bee honey (Ramos \& Cate, 1992). A similar observation has been made with Venturia canescens (Hymenoptera: Ichneumonidae) (Eliopoulos et al., 2005). Trichogramma chilonis, taxonomically much closer parasitoid showed similar results. It lived 3.60 \pm 0.26 days on $50 \%$ diluted honey (Saljoqi \& Khajjak, 2007). Normally, bee honey composed primarily of the sugars glucose $(38.5 \%)$, fructose $(38.5 \%)$ and water $(17 \%)$. The rest is sucrose $(1 \%)$, other sugars $(7 \%)$, amino acids, vitamins and minerals (National Honey Board, 2013). Different sugars in honey and amino acid and vitamin, may have positively influenced on longevity of parasitoids. 


\section{Evaluation of honey concentration suitable for adult parasitoids}

The artificial diets such as bee honey solution increase parasitoid longevity to a maximum level when provided with suitable concentrations (Idris \& Grafius, 1995). Longevity primarily depended on the concentration of the sugar solution, rather than the quantity of sugar solution ingested (Azzouz et al., 2004).

Adult longevity of $T$. bactrae significantly varied $(\mathrm{F}=6.922 \mathrm{df}=6,693 \mathrm{P}<0.001)$ among different concentrations of bee honey. The highest longevity of $7.9 \pm 0.2$ days was found when they were fed with bee honey $(50 \%)$ while the lowest longevity was $1.5 \pm 0.2$ days, when the parasitoids were fed with distilled water. Longevity of adults when there fed on 17 , $20,25,33$ and $100 \%$ were 2.2, 2.6, 3.5, 6.0 and 6.1 days respectively. A similar result was found by Leatemia et al., (1995) in which the longevity of female Trichogramma minutum Riley (Hym: Trichogrammatidae) adult had 26.5 \pm 5.4, $28.5 \pm 5.4,25.4 \pm 6.1$ and $20.7 \pm 6.1$ days longevity when adult were fed on $20,40,60$ and 80 percent bee honey respectively.

\section{Evaluation of different methods of feeding of adult parasitoids}

Adult feeding method may have an effect on access to food and convenience and safety of feeding. Of the methods used, providing honey using a sponge showed the lowest longevity, $3 \pm 0.3$ days while that of on filter paper method had the highest longevity, $8 \pm 0.3$ days. However practical usage of filter paper method is difficult in terms of labour and time consumption. Therefore it is convenient to use drop method over the filter paper method as a feeding method for parasitoids. There was a significant variation of longevity of parasitoids among the different feeding methods $(\mathrm{F}=42.74 \mathrm{df}=3,316 \mathrm{P}<0.001)$. Drop method, and wick method showed $4.5 \pm 0.3$ and $3.5 \pm 0.3$ days longevity respectively.

\section{Determination of the fecundity of the parasitoids}

Trichogrammatoidea bactrae oviposited over five days, at a decreasing rate. The rate of oviposition significantly varied with the age of the parasitoids. The highest oviposition was found in one day old parasitoids (31.2 eggs per female per day) and it was $56.05 \%$ of the total egg load. The mean number of eggs laid in the second, third, fourth and fifth days were $13.5,7.9,2.9$ and 0.4 respectively. Those represent $24,14,5$ and $0.7 \%$ of the total fecundity. These results agree with the Naranjo (1993) who reported that $T$. bactrae has higher rate of oviposition in very early in adult life. Presence of relatively high rate of oviposition soon after release may be a significant, positive feature of successful biological control agent. Mean fecundity of T. bactrae at $27{ }^{\circ} \mathrm{C}$ was 55.7 eggs per female. Our mean fecundity data of T. bactrae slightly similar to result of Naranjo (1993). Mean fecundity of T. bactrae is peaked at $25{ }^{\circ} \mathrm{C}, 55$ eggs per female (Naranjo, 1993).

\section{Determining parasitism levels over different generations}

There was a significant variation $(\mathrm{F}=5.26 \mathrm{df}=14,89 \mathrm{P}<0.001)$ of level of parasitism with the number of generation or the progeny numbers (Fig. 1). There was a general trend of loosing the ability of parasitism as the progeny continues on alternative hosts. By some reasons, level of parasitism recorded in generation one is exceptionally low, $(35.5 \%)$. Some parasitoid individuals did not oviposite at all. Upon exclusion of those, a significant variation of parasitism was found along with generation numbers. The parasitism levels steadily decrease from 66 to $35 \%$, when parasitoids reach $15^{\text {th }}$ generation from second generation. A similar 
result of parasitism has been reported and it was associated with genetic changes in the colony or adaptation to lab conditions (Hopper et al., 1993).

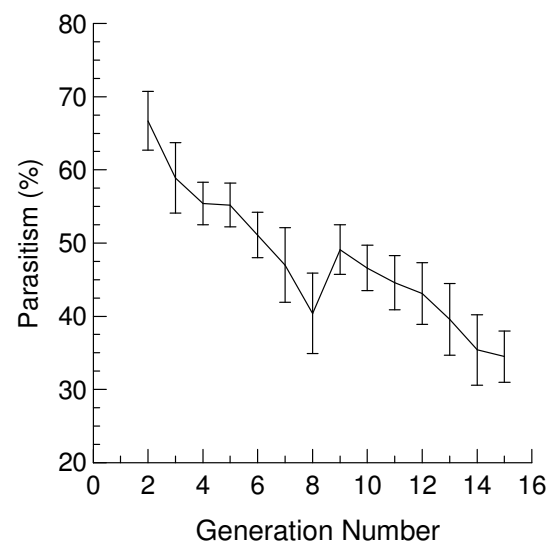

Fig 2. Level of parasitism of $T$. bactrae with the number of generation when reared on $C$. cephalonica eggs under laboratory conditions.

The successful growth, development and reproduction of insects obviously depend on their nutrition, the attainment of their qualitative and quantitative requirements. Feeding of adult parasitoids increased longevity in the laboratory. It helps to increase mass production efficiency. Hence, our findings help to maintain efficient parasitoid cultures in laboratory. Maintaining of viable and efficient parasitoid cultures is one of the important steps of augmentative release. Such information can be a basis to improving the longevity of this biocontrol agent by sugar feeding in the field and in greenhouses. Parasitoid biology related factors may be helpful for the successfully implement the biological control and mass production T. bactrae.

\section{CONCLUSIONS}

Of the food tested bee honey, glucose, fructose and sucrose; bee honey was the best for Trichogrammatoidea bactrae to keep them alive over the longest period. Fifty percent bee honey can be used as the favourable diet for T. bactrae for mass rearing. Filter paper feeding was suggested as the best feeding method, when rearing parasitoids in laboratory. However for practical usage, filter paper method is difficult in terms of labour and time consumption. T. bactrae can be successfully reared over several months (about 3 months) under laboratory conditions, but loss of parasitism is problematic. Hence, offering natural hosts for oviposition after every six generations is suggested.

\section{ACKNOWLEDGEMENT}

Financial support received through National Research Council (Grant no 11-116) is greatly appreciated. 


\section{REFERENCES}

Azzouza, B.H., Giordanengo, P., Wackersc, F.L. and Kaisera, L. (2004). Effects of feeding frequency and sugar concentration on behaviour and longevity of the adult aphid parasitoid: Aphidius ervi (Haliday) (Hymenoptera: Braconidae). Biol. Control. 31, 445 - 452.

Eliopoulos, P.A., Stathas, G.J. and Bouras, S.L. (2005). Effects and interactions of temperature, host deprivation and adult feeding on the longevity of the parasitoid Venturia canescens (Hymenoptera: Ichneumonidae). Eur. J. Entomol. 102, 181 - 187.

Elzen, G.W. and King, E.G. (1999). Periodic release and manipulation of natural enemies. pp. 253-270. In: Bellows, T.S. and Fisher, T.W. (Ed.). Handbook of Biological Control. Academic Press, San Diego, California, USA.

Greathead, D.J. and Greathead, A.H. (1992). Biological control of insect pests by insect parasitoids and predators: the BIOCAT database. Biocontrol News and Information. 13, 61 68.

Hagley, E.A.C. and Barber, D.R. (1992). Effect of food sources on the longevity and fecundity of Pholetesor ornigis (Weed) (Hymenoptera: Braconidae). Can. Entomol. 124, 341 $-346$.

Hopper, K.R., Roush, R.T. and Powell, W. (1993). Management of genetics of biologicalcontrol introductions. Ann. Rev. Entomol. 38, 27 - 51.

Idris, A.B. and Grafius, E. (1995). Wildflowers as nectar sources for Diadegma insulare (Hymenoptera: Ichneumonidae), a parasitoid of Diamondback moth (Lepidoptera: Yponomeutidae). Environ. Entomol. 24, 1726 - 1735.

Jacob, H.S. and Evans, E.W. (1998). Effects of Sugar Spray and Aphid Honeydew on Field Populations of the Parasitoid Bathyplectes curculionis (Hymenoptera: Ichneumonidae). Environ. Entomol. 27(6), 1563 - 1568.

Leatemia, J.A., Laing, J.E. and Corrigan, J.E. (1995). Effects of adult nutrition on longevity, fecundity and offspring sex ratio of Trichogramma minutum Riley (Hymenoptera: Trichogrammatidae). Can. Entomol. 127, 245 - 254.

Lee, J.C. and Heimpel, G.E. (2005). Impact of flowering buckwheat on Lepidopteran cabbage pests and their parasitoids at two spatial scales. Biol. Control. 34, 290 - 301.

Mohr, N.A. and Jay, S.C. (1990). Nectar production of selected cultivars of Brassica campestris L. and Brassica napus L. J. Apic. 29, 95 - 100.

Naranjo, S.E. (1993). Life history of Trichogrammatoidea bactrae (Hymenoptera: Trichogrammatidae), an egg parasitoid of pink bollworm (Lepidoptera: Gelechiidae), with emphasis on performance at high temperatures. Environ. Entomol. 22, 1051 - 1059.

National honey board, (2013). Honey composition [online]. [Accessed on 24.07.2013]. Available at http://www.honey.com/ 
Ramos, J.A.M. and Cate, J.R. (1992). Rate of increase and adult longevity of Catolaccus grandis (Burks) (Hymenoptera: Pteromalidae) in the laboratory of four temperatures. Environ. Entomol. 21, 620 - 627.

Saljoqi, A.U.R. and Khajjak, A.S.K. (2007). Effect of different artificial diets on the efficiency and development of Trichogramma chilonis (Ishii) (Hymenoptera: Trichogrammatidae). Sarhad J. Agric. 23, 129 - 134. 